\title{
PD-L1 biomarker testing for non-small cell lung cancer: truth or fiction?
}

\author{
Claud Grigg ${ }^{*}$ (D) and Naiyer A. Rizvi
}

\begin{abstract}
Research in cancer immunology is currently accelerating following a series of cancer immunotherapy breakthroughs during the last 5 years. Various monoclonal antibodies which block the interaction between checkpoint molecules PD-1 on immune cells and PD-L1 on cancer cells have been used to successfully treat non-small cell lung cancer (NSCLC), including some durable responses lasting years. Two drugs, nivolumab and pembrolizumab, are now FDA approved for use in certain patients who have failed or progressed on platinum-based or targeted therapies while agents targeting PD-L1, atezolizumab and durvalumab, are approaching the final stages of clinical testing. Despite impressive treatment outcomes in a subset of patients who receive these immune therapies, many patients with NSCLC fail to respond to anti-PD-1/PD-L1 and the identification of a biomarker to select these patients remains highly sought after. In this review, we discuss the recent clinical trial results of pembrolizumab, nivolumab, and atezolizumab for NSCLC, and the significance of companion diagnostic testing for tumor PD-L1 expression.
\end{abstract}

Keywords: NSCLC, PD-1, PD-L1, Immunotherapy, Nivolumab, Pembrolizumab, Immune checkpoint inhibitor, Biomarker, Lung cancer

\section{Background}

Non-small cell lung cancer (NSCLC) is by far the leading cause of cancer related mortality in the US and worldwide $[1,2]$. It is frequently diagnosed in the metastatic or unresectable setting, while those patients that do undergo potentially curative surgery will frequently relapse [3]. Despite advances in cancer treatment and survival over the last 30 years, improvements in survival for lung cancer patients have been comparatively modest, prompting research into new modalities of therapy for lung cancer [2]. The introduction of molecularly targeted agents to NSCLC therapy was a major breakthrough, though these drugs benefit only a small proportion of patients (mostly never smokers) who harbor activating genetic alterations including EGFR, ALK, and ROS1 [4].

NSCLC was not traditionally considered to be an immunotherapy responsive tumor type when the earliest clinical trials employed interleukin-2, vaccines, and interferons [5]. More recently, major treatment responses have been observed with the use of immune checkpoint inhibitors. Immune checkpoints are proteins on the

\footnotetext{
* Correspondence: cmg2204@cumc.columbia.edu

NewYork-Presbyterian/Columbia University Medical Center, Hematology/ Oncology, 177 Fort Washington Avenue, 6GN-435, New York, NY 10032, USA
}

surface of lymphocytes and other immune cells, most notably on cytotoxic T-cells. When bound to their specific ligand, often another surface bound protein on a neighboring cell, they can transmit stimulatory or inhibitory signals to activate or dampen the cellular adaptive immune response [6]. Mounting evidence suggests that the predominant mechanism by which NSCLC evades detection and elimination by the immune system is by exploiting one such inhibitory pathway through the expression of programmed death ligand 1 (PD-L1, B7-H1) [7]. PD-L1 then binds to its receptor, programmed cell death protein 1 (PD-1), on surveilling lymphocytes and initiates a signaling cascade which leads to lymphocyte exhaustion, a state of impaired function [8].

The most successful immune checkpoint inhibitors so far are monoclonal antibodies which bind to either PD-1 or PD-L1 and prevent their interaction at the tumorimmune interface. The depth and durability of responses in NSCLC have revolutionized the conceptual approach to lung cancer treatment. Still, fewer than a quarter to half of patients, even in highly selected cohorts, have experienced a clinical benefit while on anti-PD-1 or anti-PD-L1 therapies. In this review, we will describe the recent data leading to the FDA approvals of nivolumab 
(Opdivo, approved in December 2014 and March 2015 for non-squamous and squamous NSCLC in the $2^{\text {nd }}$ line setting) and pembrolizumab (Keytruda, approved in October 2015 for PD-L1 positive NSCLC also in the $2^{\text {nd }}$ line setting). We will also explore the ongoing effort and challenges to identify a PD-L1 assay that can select patients who will benefit from these drugs.

\section{Nivolumab}

Four large clinical trials have reported on the use of nivolumab for NSCLC, however some of the most important observations came from the initial phase I trial published in 2010. That trial enrolled patients with diverse malignancies, including six patients with NSCLC, but only a small number of responses were seen [9]. In several patients, pre- and post- treatment biopsies were tested for PD-L1 expression by immunohistochemistry (IHC) using a non-commercial antibody (5H1), with an indication that PD-L1 expression correlated with response. Post-treatment tumor lesions were infiltrated with CD8+ but not CD4+ lymphocytes. In the peripheral blood, T-cell markers, but not B- or NK-cell markers, were noted to drop by day 2 of treatment, but then increased substantially and remained elevated for about 30 days. These findings suggested that the effects of nivolumab treatment were rapid and resulted in a major redistribution of existing lymphocytes, followed by a prolonged period of immune activation which correlated with the anti-tumor response [9].

A much larger phase I study was reported in 2012, which included 122 evaluable patients with heavily pretreated NSCLC [10]. Treatment was well tolerated, though fatigue was common and seen in approximately $40 \%$ of patients who experienced a treatment related adverse event (AE). Serious AEs occurred in $11 \%$ of all patients including two deaths in the NSCLC cohort due to pneumonitis. These early deaths may have been preventable with earlier intervention, as the autoimmune toxicities of the drugs were still poorly understood at the time. Still, just $5 \%$ of patients discontinued treatment for toxicity reasons and some patients with immune-related endocrinopathies, colon and liver toxicities tolerated treatment re-challenge. Clinical activity was evident, with 14 responses (18\%) seen in the NSCLC group, and $3 \mathrm{mg} / \mathrm{kg}$ (every 2 weeks) was identified as the optimal dose. Of 61 pre-treatment tumors in the study tested for PD-L1 using the same $5 \mathrm{H} 1$ antibody, $36 \%$ of positive patients but none of the negative patients responded [10].

Long-term follow-up is now available from the phase I expansion, totaling 129 NSCLC patients with a median follow-up of 39 months (up to 66 months) as of 2015 [11]. All patients had failed at least one chemotherapy regimen, $54 \%$ had failed three or more. The overall response rate was $17 \%$ (and was $22 \%$ at the doses considered therapeutic:
$3 \mathrm{mg} / \mathrm{kg}$ and $10 \mathrm{mg} / \mathrm{kg}$ ) with most responses lasting over a year and the longest ongoing at 3 years. A further $5 \%$ of patients experienced an initial disease pseudo-progression or a mixed response, both phenomena consistent with an immune pattern of response that is not captured by standard Response Evaluation Criteria in Solid Tumors (RECIST) criteria [12]. Responses were seen regardless of subgroups: squamous and non-squamous, EGFR and KRAS mutated, PD-L1 positive and negative, while those with a smoking history more than 5 pack-years did much better (overall response rate [ORR] $30 \%$ vs 0 for $<5$ packyears). The median overall survival (OS) was 9.9 months, and at 3 years $27 \%$ of patients were still alive and $9 \%$ progression-free. Only six treatment related grade 3 or 4 AEs were reported including three pneumonitis, one colitis, and one hepatitis. One reassuring observation was that half of the 18 patients who discontinued therapy for toxicity had a continued response lasting $>9$ months, suggesting that early re-challenging with anti-PD-1 therapies after toxicity may not be necessary in some patients who have responded.

Three phase II and III clinical trials were initiated to evaluate squamous and non-squamous tumors independently. For squamous NSCLC, a phase II single arm study enrolled 117 heavily pre-treated patients with unresectable disease, the vast majority of whom were prior or current smokers [13]. The best ORR was $14.5 \%$, with one patient having a complete response by investigator assessment. Treatment toxicity was higher in this population, $27 \%$ of patients required a dose delay and $17 \%$ experienced a treatment related grade 3 or 4 $\mathrm{AE}$, including four patients with pneumonitis and three with colitis. Low grade gastrointestinal (GI) toxicity and fatigue were also common but manageable.

CHECKMATE 017 and CHECKMATE 057 were parallel, randomized controlled phase III trials for advanced, platinum-refractory squamous and non-squamous NSCLC, respectively, and are summarized in Table 1 . In CHECKMATE 017 [14], nivolumab bested docetaxel $\left(75 \mathrm{mg} / \mathrm{m}^{2}\right.$ every 3 weeks) in terms of the primary endpoint OS (median 9.2 vs 6.0 months; hazard ratio [HR] $0.59, p<0.001)$ and ORR (20 \% vs $9 \%$; odds ratio [OR] 2.6, $p=0.008)$. It is important to mention that $34 \%$ of patients had previously received paclitaxel, though this is unlikely to fully account for the differential activities. Consistent with the earlier studies, 9 patients on nivolumab (6.5 \%) experienced a delayed response following an initial pseudo-progression. Nivolumab was much better tolerated than docetaxel, with grade 3 or 4 AEs in just $7 \%$ of nivolumab treated patients vs $55 \%$ for docetaxel. The most common immune-related toxicities were hypothyroidism, colitis, pneumonitis, nephritis, and rash, each occurring at a rate of about 4-8 \% (all grades), and there were no immune-related deaths. 
Table 1 Response rates to anti-PD-1 and overall survival in NSCLC by study

\begin{tabular}{|c|c|c|c|c|c|c|}
\hline Study & Histology & Treatment & \# patients & ORR (\%) & $\begin{array}{l}\text { Median response } \\
\text { duration (months) }\end{array}$ & OS (months) \\
\hline \multicolumn{7}{|l|}{ Nivolumab } \\
\hline \multirow[t]{2}{*}{ Gettinger et al. [11 $]^{a}$} & \multirow[t]{2}{*}{ All } & Nivolumab 3 mg/kg q2wks & 37 & 24.3 & 17 & 14.9 \\
\hline & & Nivolumab 10 mg/kg q2wks & 59 & 20.3 & 19.1 & 9.2 \\
\hline $\begin{array}{l}\text { Rizvi et al. [13] } \\
\text { (CHECKMATE 063) }\end{array}$ & Squamous & Nivolumab 3 mg/kg q2wks & 117 & 14.5 & Not reached & 8.2 \\
\hline \multirow{2}{*}{$\begin{array}{l}\text { Brahmer et al. [14] } \\
\text { (CHECKMATE 017) }\end{array}$} & \multirow[t]{2}{*}{ Squamous } & Nivolumab 3 mg/kg q2wks & 135 & 20 & Not reached & 9.2 \\
\hline & & Docetaxel 75 mg/m² q3wks & 137 & 9 & 8.4 & 6 \\
\hline \multirow{2}{*}{$\begin{array}{l}\text { Borghaei et al. [15] } \\
\text { (CHECKMATE 057) }\end{array}$} & \multirow[t]{2}{*}{ Non-squamous } & Nivolumab 3 mg/kg q2wks & 292 & 19 & 17.2 & 12.2 \\
\hline & & Docetaxel 75 mg/m² q3wks & 290 & 12 & 5.6 & 9.4 \\
\hline Bauer et al. [55] & All & Nivolumab 3 mg/kg q 2wks & 51 & 13.7 & Not reported & Not reported \\
\hline \multicolumn{7}{|l|}{ Pembrolizumab } \\
\hline \multirow{3}{*}{$\begin{array}{l}\text { Garon et al. [16] } \\
\text { (KEYNOTE 001) }\end{array}$} & \multirow[t]{3}{*}{ All } & Pembrolizumab 2 mg/kg q3wks & 6 & 33.3 & 12.5 & 9.3 (prior therapy) \\
\hline & & Pembrolizumab 10 mg/kg q3wks & 287 & 19.2 & & 16.2 (no prior therapy) \\
\hline & & Pembrolizumab 10 mg/kg q2wks & 202 & 19.3 & & \\
\hline \multirow{3}{*}{$\begin{array}{l}\text { Herbst et al. [17] } \\
\text { (KEYNOTE 010) }\end{array}$} & \multirow[t]{3}{*}{ All } & Pembrolizumab 2 mg/kg q3wks & 344 & 18 & Not reached & 10.4 \\
\hline & & Pembrolizumab 10 mg/kg q3wks & 346 & 18.5 & Not reached & 12.7 \\
\hline & & Docetaxel 75 mg/m² q3wks & 343 & 9.3 & 6 & 8.5 \\
\hline
\end{tabular}

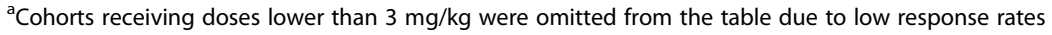

CHECKMATE 057 enrolled exclusively patients with non-squamous NSCLC and patients were randomized to docetaxel or nivolumab [15]. Again, all study outcomes favored nivolumab including OS (12.2 vs 9.4 months), ORR (19\% vs $12 \%$ ), and safety (grade 3 or 4 treatment related AEs in $10 \%$ vs $54 \%$ ) with similar rates of immune-related toxicities to CHECKMATE 017. In the subgroup analysis, no survival advantage was observed for never smokers nor for those with activating EGFR mutations. Patients receiving third line therapy or with brain metastases also did not benefit from nivolumab over docetaxel, but this was probably more reflective of an underlying aggressive or advanced disease variant than of a differential treatment effect.

\section{Pembrolizumab}

Two clinical trials have studied pembrolizumab in patients with advanced NSCLC. KEYNOTE 001 was a large phase I trial with a NSCLC expansion cohort that enrolled 495 patients who were treated with pembrolizu$\mathrm{mab} 2 \mathrm{mg} / \mathrm{kg}$ or $10 \mathrm{mg} / \mathrm{kg}$ every $2-3$ weeks [16]. The trial included 101 patients who had never received systemic therapy. Objective responses were seen in $19.4 \%$ of patients, including $24.8 \%$ of chemotherapy naïve patients, while another $21.8 \%$ of patients achieved stable disease. Treatment was effective at all tested doses and schedules, thus an every 3 week schedule was chosen for the phase III study. Responses were more common in former or current smokers compared to non-smokers (22.5\% vs $10.3 \%)$. Treatment-related adverse events were common (70.9\%), and $9.5 \%$ of patients had grade $3-5$ toxicities. Immune-related toxicities like hypothyroidism (6.9 \%) and pneumonitis (3.6\%) were mostly manageable though one patient died of severe pneumonitis. Fresh tumor biopsies were required for PD-L1 staining by IHC, and observed response rates were higher with increasing percentages of tumor PD-L1 expression (Fig. 1). Responses were durable, lasting a median of 12.5 months (range 1-23.3 months). Median OS was 9.3 months for previously treated patients and 16.2 months for chemotherapy naïve patients.

KEYNOTE 010 was a randomized phase III trial analogous to CHECKMATE 017 and 057, comparing pembrolizumab at two doses, $2 \mathrm{mg} / \mathrm{kg}$ and $10 \mathrm{mg} / \mathrm{kg}$ every 3 weeks, to docetaxel in 1034 patients [17]. The study enrolled only patients with at least $1 \% \mathrm{PD}-\mathrm{L} 1$ positive staining, with the last 593 patients stratified by PD-L1 positivity using a $50 \%$ cutoff. Patients treated with either dose of pembrolizumab had a higher median OS than with docetaxel $(10 \mathrm{mg} / \mathrm{kg}: 12.7$ months vs 8.5 months; HR 0.61, $p<0.0001 ; 2 \mathrm{mg} / \mathrm{kg}: 10.4$ months, HR 0.71, $p=0.0008$ ). There was a non-significant trend toward longer survival in the $10 \mathrm{mg} / \mathrm{kg}$ cohort compared with the $2 \mathrm{mg} / \mathrm{kg}$ cohort. When stratified by PD-L1 positivity, defined as a proportion score (PS) $\geq 50 \%$ staining of the tumor, the survival benefits were more pronounced (HR 0.50 and 0.54 for $10 \mathrm{mg} / \mathrm{kg}$ and $2 \mathrm{mg} / \mathrm{kg}$ cohorts, respectively), with median survivals of 17.3 and 14.9 months. In the subgroup analysis, both squamous and non-squamous histology favored pembrolizumab treatment, consistent with 


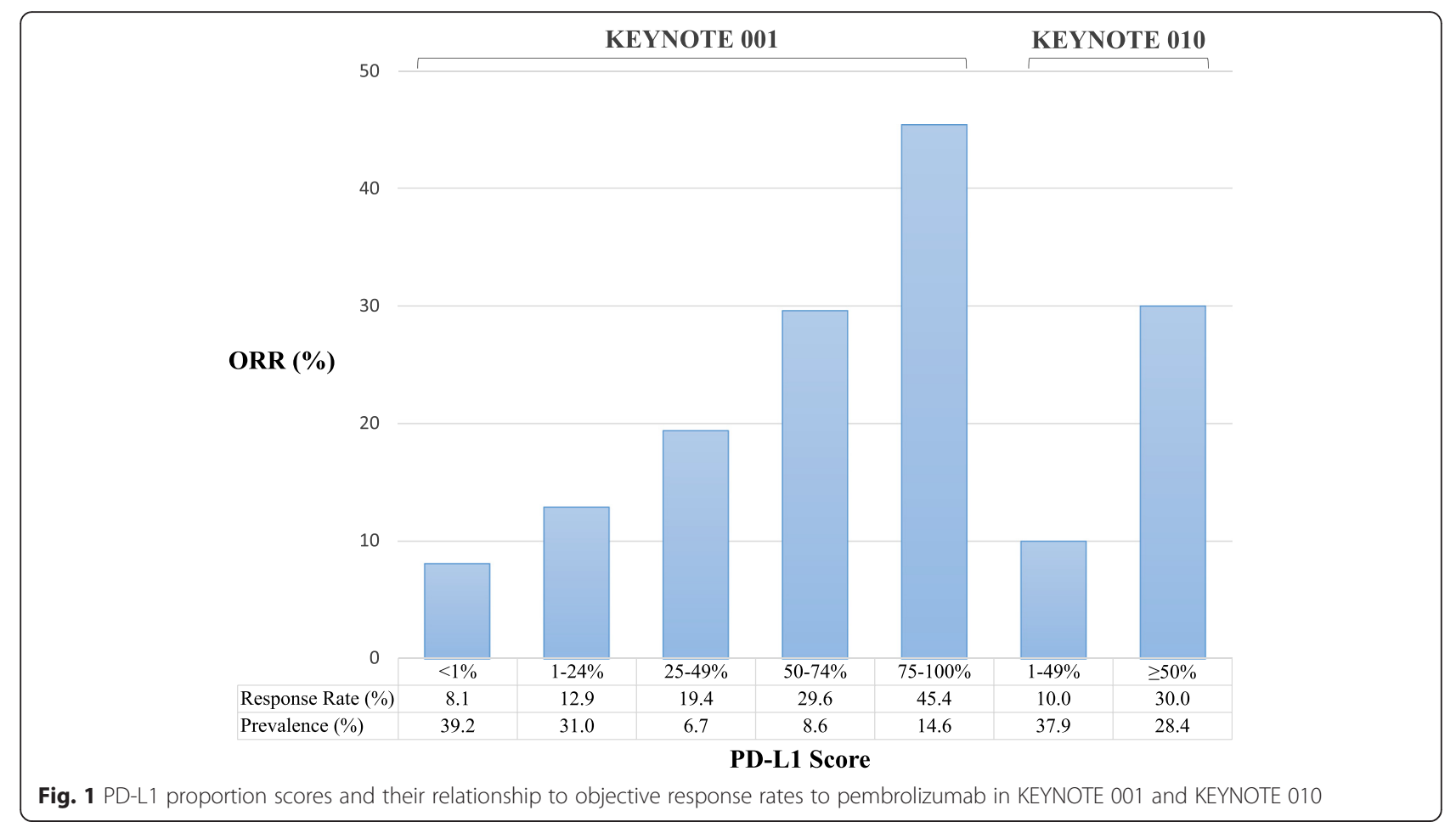

results from the nivolumab clinical trials. Similarly, EGFR mutated tumors seemed to have no survival advantage with pembrolizumab over docetaxel, though the number of patients $(n=86)$ was small. Pembrolizumab was more tolerable than docetaxel, with fewer grade 3-5 AEs (14\% vs $35 \%$ ) and fewer drug discontinuations ( $4.7 \%$ vs $10 \%$ ). There were six $(0.9 \%)$ pembrolizumab-attributed deaths, three due to pneumonitis, two pneumonia, and one myocardial infarction, and five (1.6\%) docetaxel-attributed deaths. Based on the unprecedented survival achieved in the PD-L1+ population, the FDA approved pembrolizumab for second line therapy but only for tumors with a PD-L1 PS $\geq 50 \%$.

\section{Companion PD-L1 testing \\ Nivolumab/Dako IHC 28-8 pharmDx}

In the multi-tumor phase I trial, a non-commercial anti-PD-L1 murine monoclonal antibody $(5 \mathrm{H} 1)$ was used to measure PD-L1 expression by IHC in tumor biopsies $[9,18]$. Each stained tumor section was scored by the degree of membranous staining on tumor cells, with a minimum requirement of 100 evaluable tumor cells. The "positive" threshold was set at $5 \%$ of cells based on test performance characteristics. There appeared to be differential response rates, with $36 \%$ of PD-L1 positive vs 0 PD-L1 negative tumors experiencing a treatment response [10]. The antibody used in this assay was later abandoned in favor of commercial assay developed by Dako using clone 28-8, a rabbit anti-human PD-L1 (see Table 2). Two of the four subsequent publications using this assay in
NSCLC have demonstrated an apparent value for this PDL1 test. In the smaller CHECKMATE 063, a single arm study in squamous NSCLC, $24 \%$ of PD-L1 positive patients $(n=25)$ and $14 \%$ of negative patients $(n=51)$ had an objective response. In CHECKMATE 057, PD-L1 positivity at $\geq 5 \%$ strongly correlated with objective response (34\% vs $14 \%$ for PD-L1 negative) as well as predicted an OS benefit compared with docetaxel (PD-L1+ HR 0.43 vs PD-L1- HR 1.01; $p<0.001$ ) [15]. Meanwhile, in CHECKMATE 017, PD-L1 positivity at any cutoff was not significantly prognostic nor predictive of benefit in squamous histology [14]. However, the power of this study was limited by a smaller sample size than CHECKMATE 057, and a closer inspection of the study outcomes suggests not only trends toward improved ORR and OS for PD-L1 positive patients, but also nearly double the number of patients in the "tail" of the PD-L1 positive progression-free survival (PFS) curve suggesting a higher likelihood of long term benefit. Most importantly, in all studies a significant proportion of PD-L1 negative patients clearly benefitted from treatment with nivolumab. Similar observations have been made in the melanoma clinical trials using the same assay (see Table 3). As a consequence, the FDA label for nivolumab did not specify any threshold PD-L1 positivity, in fact it did not require PD-L1 testing at all and the Dako 28-8 assay was labelled as "complementary."

\section{Pembrolizumab/Dako IHC 22C3 pharmDx}

The pembrolizumab companion PD-L1 assay, also commercially available from Dako, uses a distinct antibody clone, 
Table 2 Companion PD-L1 Assays in Development for PD-1/PD-L1 Inhibitors

\begin{tabular}{|c|c|c|c|c|}
\hline Drug & Drug target & Companion antibody clone & Developer & Definition of positive test \\
\hline \multirow[t]{2}{*}{ Nivolumab } & \multirow[t]{2}{*}{ PD-1 } & \multirow[t]{2}{*}{$28-8$} & Dako & \multirow{2}{*}{$\begin{array}{l}\geq 5 \% \text { membranous staining of tumor cells } \\
\text { (minimum } 100 \text { cells evaluated) }\end{array}$} \\
\hline & & & Bristol-Meyers Squibb & \\
\hline \multirow[t]{2}{*}{ Pembrolizumab } & \multirow[t]{2}{*}{ PD-1 } & \multirow[t]{2}{*}{$22 \mathrm{C} 3$} & Dako & \multirow{2}{*}{$\begin{array}{l}\geq 1 \%^{\mathrm{a}} \text { membranous staining of tumor cells } \\
\text { or immune cells that are intercalating or at } \\
\text { the tumor interface }\end{array}$} \\
\hline & & & Merck & \\
\hline \multirow[t]{6}{*}{ Atezolizumab (MPDL3280A) } & \multirow[t]{6}{*}{ PD-L1 } & \multirow[t]{6}{*}{ SP142 } & Ventana & \multirow{2}{*}{$\begin{array}{l}\text { Each specimen assigned a score based on } \\
\text { both tumor and immune cell PD-L1: }\end{array}$} \\
\hline & & & \multirow[t]{5}{*}{ Genentech/Roche } & \\
\hline & & & & TC3/IC3 PD-L1 $\geq 50 \%$ \\
\hline & & & & TC2/IC2 PD-L1 5-49 \% \\
\hline & & & & TC1/IC1 PD-L1 1-4 \% \\
\hline & & & & TCO/ICO PD-L1 < $1 \%$ \\
\hline \multirow[t]{2}{*}{ Durvalumab (MED/4736) } & \multirow[t]{2}{*}{ PD-L1 } & \multirow[t]{2}{*}{ SP263 } & Ventana & \multirow[t]{2}{*}{$\geq 25 \%$ membranous staining of tumor cells } \\
\hline & & & Medlmmune/AstraZeneca & \\
\hline
\end{tabular}

${ }^{\mathrm{a}}$ The FDA indication in NSCLC for pembrolizumab requires PS $\geq 50 \%$

22C3 (mouse anti-human PD-L1). This automated assay is performed on formalin-fixed, paraffin-embedded (FFPE) tissue using similar conditions for heat-based antigen retrieval and staining as the 28-8 assay. After staining is completed, the percentage of membranous PD-L1 staining of neoplastic or intercalating immune cells is counted manually and the PS is reported as a percentage (see Table 2).

In KEYNOTE 001 for NSCLC, it was evident early in the trial that increasing efficacy correlated with PD-L1 positivity by this IHC assay [19]. Following enrollment of 51 patients, the study was modified to include only patients with at least $1 \%$ PD-L1 positivity [16]. The investigators also noted that when archival tissue over 6 months old was used for testing, the PD-L1 protein had deteriorated resulting in unreliable staining. To identify an optimal cutoff for PD-L1 positivity, a training cohort of 61 tumors was stained for PD-L1 and a threshold PS $\geq 50 \%$ was established as the positive threshold. Among the total screened patients, the prevalence of PD-L1 PS $\geq 50 \%$ was $23.2 \%$, while another $37.6 \%$ had a PS between 1 and $49 \%$. Patients with activating EGFR mutations or ALK rearrangement were equally as likely to have high PD-L1 expression as non-mutated tumors, though the total patients with these mutations was low.

At the time of analysis, both PFS and OS were considerably longer for the group with a PD-L1 PS $\geq 50 \%(\sim 40$ and $65 \%$ at 1 year, respectively), while PFS and OS were similar for the groups with a PS $\leq 1 \%$ or $1-49 \%(\sim 10$ and $40 \%$ at 1 year). The duration of response, however, was no different between groups, suggesting that even patients with PD-L1 "negative" tumors could attain a durable, meaningful benefit albeit at a much lower frequency than the PD-L1 "positive" tumors.

In KEYNOTE 010 the same assay was used with a threshold for PD-L1 high (PS $\geq 50 \%$ ), intermediate (PS 1$49 \%$ ), or low (PS $\leq 1 \%$ ) tumors, roughly a third of patients fell into each category and those with PS $\leq 1 \%$ were excluded from the trial. As noted earlier in this review, patients with a higher PS were much more likely to have an objective response to pembrolizumab (30 \%), however responses were still observed in $10 \%$ of those with a PS 1$49 \%$ and the OS subgroup analysis still favored pembrolizumab over docetaxel (HR 0.76, 95 \% CI 0.60-0.96). Interestingly, this group with intermediate PD-L1 expression did not have a PFS advantage over docetaxel (HR 1.04), a potential indicator that atypical immunologic anti-tumor responses are more common in this subset.

\section{Other antibodies in development}

Several anti-PD-1 (pidilizumab/CT-011, REGN2810) and anti-PD-L1 antibodies (durvalumab/MEDI4736, atezolizumab/MPDL3280A, avelumab/MSB0010718C, BMS-936559) are in various stages of clinical development for NSCLC and other cancers. Like nivolumab and pembrolizumab, these agents are designed to block the interaction of PD-1 with PD-L1 and most have been modified to have no Fcmediated antibody dependent cellular cytotoxicity.

A phase II randomized trial (POPLAR) with atezolizumab was recently published. In this trial, 287 patients with previously treated advanced or metastatic NSCLC were randomized 1:1 to docetaxel or atezolizumab (given at a flat dose of $1200 \mathrm{mg}$ IV every 3 weeks) [20]. Overall survival, the primary endpoint, was improved in the atezolizumab arm by nearly 3 months (median OS 12.6 months vs 9.7 months; HR 0.73, $p=0.04$ ), while safety was similar to other anti-PD-1 agents (11\% with treatment related grade 3 or 4 AEs). Responses lasted a median of 14.3 months (vs 7.2 months for docetaxel), while neither the ORR nor PFS were higher in the atezolizumab arm, confirming that traditional radiographic criteria are imprecise measures of benefit from immunotherapy. 
Table 3 Response rates to anti-PD-1/PD-L1 antibodies in NSCLC and selected malignancies according to PD-L1 positivity

\begin{tabular}{|c|c|c|c|c|c|}
\hline Study & Antibody & Tumor type & PD-L1 cutoff & $N$ & Response (\%) \\
\hline \multicolumn{6}{|l|}{ Nivolumab } \\
\hline \multirow[t]{2}{*}{ Topalian et al. [10] } & $5 \mathrm{H} 1$ & Multiple & $\geq 5 \%$ & 25 & 36 \\
\hline & & & $<5 \%$ & 17 & 0 \\
\hline \multirow[t]{2}{*}{ Gettinger et al. [11] } & $28-8$ & NSCLC & $\geq 5 \%$ & 33 & 15 \\
\hline & & & $<5 \%$ & 35 & 14 \\
\hline \multirow[t]{2}{*}{ Rizvi et al. [13] } & $28-8$ & Squamous NSCLC & $\geq 5 \%$ & 25 & 24 \\
\hline & & & $<5 \%$ & 51 & 14 \\
\hline \multirow[t]{2}{*}{ Brahmer et al. [14] } & $28-8$ & Squamous NSCLC & $\geq 5 \%$ & 42 & 21 \\
\hline & & & $<5 \%$ & 75 & 15 \\
\hline \multirow[t]{2}{*}{ Borghaei et al. [15] } & $28-8$ & Non-squamous NSCLC & $\geq 5 \%$ & 95 & 34 \\
\hline & & & $<5 \%$ & 136 & 14 \\
\hline \multirow[t]{2}{*}{ Hodi et al. [56] } & $28-8$ & Melanoma & $\geq 5 \%$ & 18 & 44 \\
\hline & & & $<5 \%$ & 23 & 13 \\
\hline \multirow[t]{2}{*}{ Robert et al. [57] } & $28-8$ & Melanoma & $\geq 5 \%$ & 74 & 53 \\
\hline & & & $<5 \%$ or indet & 136 & 33 \\
\hline \multirow[t]{2}{*}{ Weber et al. [58] } & $28-8$ & Melanoma (ipilimumab refractory) & $\geq 5 \%$ & 55 & 44 \\
\hline & & & $<5 \%$ & 64 & 20 \\
\hline \multirow[t]{2}{*}{ Larkin et al. [59] } & $28-8$ & Melanoma & $\geq 5 \%$ & 80 & 58 \\
\hline & & & $<5 \%$ & 208 & 41 \\
\hline \multicolumn{6}{|l|}{ Pembrolizumab } \\
\hline \multirow[t]{2}{*}{ Garon et al. [16] } & $22 \mathrm{C} 3$ & NSCLC & $\geq 50 \%$ & 119 & 41 \\
\hline & & & $<50 \%$ & 237 & 13 \\
\hline \multirow[t]{2}{*}{ Herbst et al. [17] } & $22 \mathrm{C} 3$ & NSCLC & $\geq 50 \%$ & 290 & 30 \\
\hline & & & $1-49 \%$ & 400 & 10 \\
\hline \multirow[t]{2}{*}{ Kefford et al. [60] } & $22 \mathrm{C} 3$ & Melanoma & $\geq 1 \%$ & 55 & 51 \\
\hline & & & $<1 \%$ & 16 & 6 \\
\hline \multirow[t]{2}{*}{ Puzanov et al. [61] } & $22 \mathrm{C} 3$ & Melanoma (ipilimumab refractory) & $\geq 1 \%$ & 193 & 26 \\
\hline & & & $<1 \%$ & 93 & 15 \\
\hline \multirow[t]{2}{*}{ Robert et al. [62] } & $22 \mathrm{C} 3$ & Melanoma & $\geq 1 \%$ & 896 & NR (PFS HR 0.53) \\
\hline & & & $<1 \%$ & 197 & NR (PFS HR 0.67-0.76) \\
\hline \multicolumn{6}{|c|}{ Atezolizumab (MPDL3280A) } \\
\hline \multirow[t]{2}{*}{ Spigel et al. [63] } & SP142 & NSCLC & TC or IC $\geq 50 \%$ & 53 & 26 \\
\hline & & & TC or IC 5-49\% & 84 & 14 \\
\hline \multirow[t]{3}{*}{ Spira et al. [20, 64] } & SP142 & NSCLC & TC or IC $\geq 50 \%$ & 24 & 38 \\
\hline & & & TC or IC 1-49\% & 69 & 12 \\
\hline & & & TC and IC $<1 \%$ & 51 & 8 \\
\hline \multirow[t]{2}{*}{ Besse et al. [65] } & SP142 & NSCLC & $\mathrm{TC}$ or $\mathrm{IC} \geq 50 \%$ & 302 & 26 \\
\hline & & & TC or IC 5-49\% & 357 & 10 \\
\hline \multirow[t]{2}{*}{ Powles et al. [66] } & SP142 & Urothelial & TC or IC $\geq 5 \%$ & 30 & 43 \\
\hline & & & TC and IC $<5 \%$ & 35 & 11 \\
\hline \multirow[t]{3}{*}{ Rosenberg et al. [67] } & SP142 & Urothelial & TC or IC $\geq 5 \%$ & 100 & 26 \\
\hline & & & TC and IC $1-5 \%$ & 107 & 11 \\
\hline & & & TC and IC $<1 \%$ & 103 & 8 \\
\hline Herbst et al. [21] & SP142 & Multiple & $\mathrm{TC}$ or $\mathrm{IC} \geq 50 \%$ & 33 & 46 \\
\hline
\end{tabular}


Table 3 Response rates to anti-PD-1/PD-L1 antibodies in NSCLC and selected malignancies according to PD-L1 positivity (Continued)

\begin{tabular}{|c|c|c|c|c|c|}
\hline & & & TC or IC 1-49\% & 57 & 19 \\
\hline & & & TC and IC $<1 \%$ & 60 & 13 \\
\hline \multicolumn{6}{|c|}{ Durvalumab (MEDI4736) } \\
\hline \multirow[t]{2}{*}{ Rizvi et al. [68] } & SP263 & NSCLC & $\geq 25 \%$ & 84 & 27 \\
\hline & & & $<25 \%$ & 92 & 5 \\
\hline \multirow[t]{2}{*}{ Segal et al. [69] } & SP263 & HNSCC & $\geq 25 \%$ & 22 & 18 \\
\hline & & & $<25 \%$ & 37 & 8 \\
\hline \multicolumn{6}{|c|}{ Avelumab (MSB0010718C) } \\
\hline \multirow[t]{2}{*}{ Gulley et al. [70] } & $?$ & NSCLC & $\geq 5 \%$ & 122 & 15 \\
\hline & & & $<5 \%$ & 20 & 10 \\
\hline \multirow[t]{2}{*}{ Apolo et al. [71] } & $?$ & Urothelial & $\geq 5 \%$ & 10 & 40 \\
\hline & & & $<5 \%$ & 22 & 9 \\
\hline
\end{tabular}

Importantly, enrollment was stratified by PD-L1 expression using a novel IHC assay (Ventana SP142, Table 3) in which PD-L1 positivity was categorized according to the expressing cell type (tumor cell [TC] or immune cell $[\mathrm{IC}]$ ) and then scored along a gradient (<1 \% [TC0 or IC0], $1-4 \%$ [TC1 or IC1], 5-49\% [TC2 or IC2], and $\geq 50 \%$ (TC3 or IC3]). Treatment with atezolizumab was favored in all but the least PD-L1 positive tumors (TC0 and IC0; HR 1.04). Other biomarkers were explored, including IHC expression of PD-L2, B7.1 (an alternative receptor for PD-L1), and PD-1 as well as an expression panel of T-effector and interferon- $\gamma$ associated genes, all of which were predictive of a survival benefit from atezolizumab.

\section{PD-L1 testing limitations}

As outlined, considerable effort has been invested to develop quantifiable, reproducible PD-L1 assays to predict which patients should receive immune checkpoint inhibitors. The commercial complementary PD-L1 diagnostic test for nivolumab (Dako 28-8 pharmDx) and companion test for pembrolizumab (Dako 22C3 pharmDx) are now FDA approved for use in NSCLC, while the complementary test for atezolizumab (Ventana SP142) is approved for urothelial carcinoma. The performance characteristics of the companion tests for atezolizumab and durvalumab (Ventana SP263) are still being assessed in NSCLC (Table 3). It is expected that each of these drugs and assays will ultimately become available to practicing oncologists.

Still, the performance of these IHC-based assays has been somewhat disappointing, as most "positive" cutoffs would exclude a considerable number of responders in the range of $10-20 \%$. Therein lies the first drawback to PD-L1 testing, which is, how is PD-L1 "positivity" defined? PD-L1 can be expressed by both tumor and inflammatory cells within the tumor microenvironment, though the relative importance of either is unclear [21]. There is no consensus to the relevance of geographic patterns of expression (eg. proximity of PD-L1 to immune infiltrating lymphocytes, membranous vs cytoplasmic) and quantitative cutoffs have been variably described. Based on the findings in KEYNOTE 001 (Fig. 1), it may make more sense to consider PD-L1 expression as a continuous measures rather than a binary "positive" or "negative." Along these lines, currently published tissue studies have found PD-L1 positivity to indicate favorable, unfavorable, or have no relationship to prognosis, as well as variable correlations with histology and mutation status in NSCLC and other tumor types [22-32].

Technical aspects of the assays are also an important source of inconsistency. This topic was reviewed in detail recently $[33,34]$. The quality of commercially available antibodies is a major concern with considerable variability in staining intensity and patterns found between antibodies [23]. Until recently, no information was available to compare the companion diagnostics in development by Dako and Ventana (Table 2). Assay variables pertaining to tissue fixation, storage, and antigen retrieval can result in PD-L1 degradation and these variables are not standardized. Importantly, the KEYNOTE 010 trial confirmed that archival tissue (as opposed to fresh tissue) can be used for PD-L1 staining and this has been observed in other trials [17, 35]. But the lack of methodologic transparency and standardization across platforms may partially explain why PD-L1 positivity has had such widely varying clinical significance, not only in NSCLC but also in other tumor types. Table 3 lists response rates for anti-PD-1 and anti-PD-L1 treatments according to companion PD-L1 "positivity" in NSCLC and selected other cancers trials.

To address these concerns, a consortium of drug manufacturers and representatives from Dako and Ventana, organized in part through a joint effort of the FDA, 
AACR, ASCO, and the International Association for the Study of Lung Cancer (IASLC), was formed with the task of creating a resource to compare the performances of the four major PD-L1 companion assays. The ultimate goal of this collaboration is to establish cross-platform standards for PD-L1 positivity analogous to those for ER, PR, and HER2 testing. Results of the pilot phase of this "Blueprint project" were presented at the AACR Annual Meeting in 2016, and included 39 lung cases reviewed by 3 pathologists [36]. Tumor cell PD-L1 staining was similar for the Ventana SP263 and two Dako assays, with less tumor staining by the Ventana SP142 assay. Interobserver variability was high when quantifying immune cell positivity but not tumor cell positivity. The performance of these assays (using their respective PD-L1 staining thresholds) varied substantially, with the Ventana SP142 assay labeling the highest number of samples as positive (79 \%) and the Ventana SP263 labeling the fewest as positive (53\%). Both Dako assays (28-8 and 22C3) performed nearly identically with an intermediate rate of positivity, and there was incomplete overlap between all of the assays. These important preliminary observations highlight the potential for false positive and negative results based solely on the assay chosen. Additional predictive information, larger sample sizes, and potentially adjustments to the cutoffs will be required before any conclusions can be made about the merits of each assay.

\section{Additional challenges to biomarker development}

There are other biological limitations to PD-L1 detection in tumor biopsies. Most traditional cancer biomarkers evaluate fixed elements that do not vary tremendously with time such as gene mutations or proteins directly involved in cellular growth signaling or replication. Examples include estrogen and progesterone receptor expression, HER2 gene amplification, or mutations in EGFR, KRAS, etc. Alternatively, the PD-L1 gene is not typically mutated or amplified and its expression is dynamic both in space and over time in response to a constantly evolving immune response. There is concern that sampling error could result in false negative tests, though some recent case series have suggested a reasonable concordance between both synchronous (same time but different location) and metachronous (different time) specimen in the range of 75-90 \% [37-40]. PD-L1 expression is also affected by concurrent or prior treatments, including radiation or chemotherapy, which may have been administered after a biopsy was obtained [41-44].

Alternative biomarker approaches have focused on quantifying and qualifying tumor infiltrating lymphocytes (TILs) or on identifying tumor neoantigens, which are fragments of mutated proteins displayed in the major histocompatibility complexes (MHC) of tumor cells and which are probably critical to the anti-tumor immune response. Several studies now have found that the mutational load, as well as the number of predicted neoantigens (according to computerized algorithms which account for $\mathrm{MHC}$ binding), are better predictors of response to checkpoint inhibition than PD-L1 IHC, TILs, or clinical variables [45-47]. While efforts are underway to better understand and identify these neoantigens through exome sequencing, surrogate measures of the mutation burden such as chronic carcinogen exposure (eg. tobacco, ultraviolet light) and defects in DNA repair mechanisms (microsatellite instability/mismatch repair defects, BRCA and POLE mutations) have emerged as clinically useful biomarkers [46-48].

\section{Conclusions}

The recent FDA approvals of nivolumab and pembrolizumab represent the earliest phase of a major paradigm shift in treating NSCLC. So far, it appears that both antiPD-1 antibodies, as well as the therapeutic anti-PD-L1 antibodies in development, will have comparable efficacy and toxicity, with responses in approximately 15-20\% of unselected NSCLC patients and serious autoimmune toxicities in 5-10\% of patients. In the absence of headto-head to comparisons or clear biological differences between these agents, it is not possible to recommend one treatment over another.

Due to the low toxicity of checkpoint inhibitors and the theorized synergy with other treatment modalities [49, 50], combination clinical trials of anti-PD-1 antibodies with chemotherapy, radiation, and other immunotherapies are ongoing in the metastatic setting. The most mature of these have combined anti-PD-1 or anti-PD-L1 antibodies with anti-CTLA-4 antibodies with impressive results at the cost of increased toxicity [51-54]. This strategy has been effective in the treatment of melanoma. It also remains to be proven that these agents are as effective in the first-line setting, though in the treatment naive cohort in KEYNOTE 001 pembrolizumab appeared equally efficacious and rates of PD-L1 positivity were comparable to previously treated patients ( $\sim 23 \%$ with PD-L1 PS $\geq 50 \%$ in either group).

In the current clinical realm of FDA-approved immunotherapies, the role of PD-L1 testing is largely prognostic for patients beginning treatment with either nivolumab or pembrolizumab given that none of the assays can conclusively identify non-benefiting patients. In light of the preliminary results from Blueprint, it is not possible to recommend one assay over another. From a practical standpoint some clinicians may choose the 22C3-based assay to have optional access to pembrolizumab in the event of a positive assay. Meanwhile many laboratories have begun reporting both tumor and immune cell positivity analogous to the atezolizumab companion assay by Ventana, however the meaning of these separate scores is still very much uncertain. As more treatment options and 
combinations become available, measures of immune activation including PD-L1 expression will clearly become more relevant, particularly as the clinician factors the line of therapy and weighs the potential benefit and toxicity of combination immunotherapy in PD-L1 positive versus negative tumors. Specifically, it remains to be shown whether PD-L1 negative patients should be treated concurrently with an anti-CTLA-4 antibody (such as ipilimumab or tremelimumab) as appears to be the evolving strategy for melanoma. It seems PD-L1 expression represents one lens and sensitivity and specificity is likely to be more robust as multiple lenses are used in the analyses.

\begin{abstract}
Abbreviations
AE, adverse event; FFPE, formalin-fixed, paraffin-embedded; GI, gastrointestinal; $H R$, hazard ratio; IC, immune cell; IHC, immunohistochemistry; MHC, major histocompatibility complexes; NSCLC, non-small cell lung cancer; OR, odds ratio; ORR, overall response rate; OS, overall survival; PD-1, programmed cell death protein 1; PD-L1, programmed death ligand 1; PFS, progression-free survival; PS, proportion score; RECIST, response evaluation criteria in solid tumors; TC, tumor cell; TILs, tumor infiltrating lymphocytes
\end{abstract}

\section{Acknowledgements}

No additional acknowledgements other than those listed as authors.

\section{Funding}

There were no sources of funding for this review.

Availability of data and materials

Not applicable.

\section{Authors' contributions}

Both authors contributed equally to the preparation of this review manuscript. Both authors read and approved the final manuscript.

\section{Authors' information}

CG is currently a Postdoctoral Clinical Fellow in Hematology and Oncology at Columbia University Medical Center with a research interest in thoracic oncology and the application of novel immunotherapies. NR is a Professor of Medicine at Columbia University Medical Center where he serves as the Director of Thoracic Oncology and Phase I Immunotherapeutics. He has been senior author and principal investigator on many clinical trials in the clinical development of multiple immune checkpoint inhibitors for NSCLC. $\mathrm{He}$ is the lead researcher on the Thoracic Oncology medical team.

\section{Competing interests}

Claud Grigg has no competing interests to report.

Naiyer Rizvi has received personal fees from Bristol-Myers Squibb, Genentech, Roche, Novartis, AstraZeneca/ Medlmmune, and Merck Sharp \& Dohme.

\section{Consent for publication}

Not applicable.

Ethical approval and consent to participate Not applicable.

Received: 11 April 2016 Accepted: 21 July 2016

Published online: 16 August 2016

\section{References}

1. Ferlay J, Soerjomataram I, Dikshit R, et al. Cancer incidence and mortality worldwide: Sources, methods and major patterns in GLOBOCAN 2012. International Journal of Cancer. 2015;136:E359-86.

2. Siegel RL, Miller KD, Jemal A. Cancer statistics, 2016. CA Cancer J Clin. 2016;66:7-30.

3. Groome PA, Bolejack V, Crowley JJ, et al. The IASLC Lung Cancer Staging Project: validation of the proposals for revision of the $\mathrm{T}, \mathrm{N}$, and $\mathrm{M}$ descriptors and consequent stage groupings in the forthcoming (seventh) edition of the TNM classification of malignant tumours. J Thorac Oncol. 2007;2:694-705.

4. Chan BA, Hughes BG. Targeted therapy for non-small cell lung cancer: current standards and the promise of the future. Transl Lung Cancer Res. 2015;4:36-54.

5. Al-Moundhri M, O'Brien M, Souberbielle BE. Immunotherapy in lung cancer. British Journal of Cancer. 1998;78:282-8.

6. Brahmer JR, Pardoll DM. Immune checkpoint inhibitors: making immunotherapy a reality for the treatment of lung cancer. Cancer Immunology Research. 2013;1:85-91.

7. Taube JM, Klein A, Brahmer JR, et al. Association of PD-1, PD-1 ligands, and other features of the tumor immune microenvironment with response to anti-PD-1 therapy. Clin Cancer Res. 2014;20:5064-74.

8. Jiang Y, Li Y, Zhu B. T-cell exhaustion in the tumor microenvironment. Cell Death Dis. 2015;6:e1792.

9. Brahmer JR, Drake CG, Wollner I, et al. Phase I study of single-agent antiprogrammed death-1 (MDX-1106) in refractory solid tumors: safety, clinical activity, pharmacodynamics, and immunologic correlates. J Clin Oncol. 2010;28:3167-75.

10. Topalian SL, Hodi FS, Brahmer JR, et al. Safety, activity, and immune correlates of anti-PD-1 antibody in cancer. N Engl J Med. 2012;366:2443-54.

11. Gettinger SN, Horn L, Gandhi L, et al. Overall survival and long-term safety of nivolumab (anti-programmed death 1 antibody, BMS-936558, ONO-4538) in patients with previously treated advanced Non-small-cell lung cancer. J Clin Oncol. 2015;33:2004-12.

12. Wolchok JD, Hoos A, O'Day S, et al. Guidelines for the evaluation of immune therapy activity in solid tumors: immune-related response criteria. Clinical Cancer Research. 2009:15:7412-20.

13. Rizvi NA, Mazieres J, Planchard D, et al. Activity and safety of nivolumab, an anti-PD-1 immune checkpoint inhibitor, for patients with advanced, refractory squamous non-small-cell lung cancer (CheckMate 063): a phase 2, single-arm trial. Lancet Oncol. 2015;16:257-65.

14. Brahmer J, Reckamp KL, Baas P, et al. Nivolumab versus docetaxel in advanced squamous-cell non-small-cell lung cancer. New England Journal of Medicine. 2015;373:123-35.

15. Borghaei H, Paz-Ares L, Horn L, et al. Nivolumab versus docetaxel in advanced nonsquamous non-small-cell lung cancer. N Engl J Med. 2015;373:1627-39.

16. Garon EB, Rizvi NA, Hui R, et al. Pembrolizumab for the treatment of non-smallcell lung cancer. The New England Journal of Medicine. 2015:372:2018-28.

17. Herbst RS, Baas P, Kim DW, et al. Pembrolizumab versus docetaxel for previously treated, PD-L1-positive, advanced non-small-cell lung cancer (KEYNOTE-010): a randomised controlled trial. Lancet. 2016;387:1540-50.

18. Thompson RH, Kuntz SM, Leibovich BC, et al. Tumor B7-H1 is associated with poor prognosis in renal cell carcinoma patients with long-term followup. Cancer Res. 2006:66:3381-5.

19. Gandhi L, Balmanoukian A, Hui R, et al. Abstract CT105: MK-3475 (anti-PD-1 monoclonal antibody) for non-small cell lung cancer (NSCLC): Antitumor activity and association with tumor PD-L1 expression. Cancer Research. 2014:74:CT105.

20. Fehrenbacher L, Spira A, Ballinger M, et al. Atezolizumab versus docetaxel for patients with previously treated non-small-cell lung cancer (POPLAR): a multicentre, open-label, phase 2 randomised controlled trial. Lancet. 2016.

21. Herbst RS, Soria JC, Kowanetz M, et al. Predictive correlates of response to the anti-PD-L1 antibody MPDL3280A in cancer patients. Nature. 2014;515:563-7.

22. $\mathrm{Mu} \mathrm{CY}$, Huang JA, Chen Y, Chen C, Zhang XG. High expression of PD-L1 in lung cancer may contribute to poor prognosis and tumor cells immune escape through suppressing tumor infiltrating dendritic cells maturation. Medical Oncology. 2011;28:682-8

23. Velcheti V, Schalper KA, Carvajal DE, et al. Programmed death ligand-1 expression in non-small cell lung cancer. Lab Invest. 2014;94:107-16.

24. Gao Q, Wang XY, Qiu SJ, et al. Overexpression of PD-L1 significantly associates with tumor aggressiveness and postoperative recurrence in human hepatocellular carcinoma. Clin Cancer Res. 2009:15:971-9.

25. Yang CY, Lin MW, Chang YL, Wu CT, Yang PC. Programmed cell deathligand 1 expression in surgically resected stage I pulmonary adenocarcinoma and its correlation with driver mutations and clinical outcomes. Eur J Cancer. 2014;50:1361-9.

26. D'Incecco A, Andreozzi M, Ludovini V, et al. PD-1 and PD-L1 expression in molecularly selected non-small-cell lung cancer patients. British Journal of Cancer. 2015;112:95-102.

27. Azuma K, Ota K, Kawahara A, et al. Association of PD-L1 overexpression with activating EGFR mutations in surgically resected nonsmall-cell lung cancer. Ann Oncol. 2014;25:1935-40 
28. Chen YB, Mu CY, Huang JA. Clinical significance of programmed death-1 ligand-1 expression in patients with non-small cell lung cancer: a 5-yearfollow-up study. Tumori. 2012;98:751-5.

29. Wang A, Wang HY, Liu Y, et al. The prognostic value of PD-L1 expression for nonsmall cell lung cancer patients: a meta-analysis. Eur J Surg Oncol. 2015;41:450-6.

30. Pan ZK, Ye F, Wu X, An HX, Wu JX. Clinicopathological and prognostic significance of programmed cell death ligand1 (PD-L1) expression in patients with non-small cell lung cancer: a meta-analysis. Journal of Thoracic Disease. 2015;7:462-70.

31. Zhong A, Xing Y, Pan X, Shi M, Xu H. Prognostic value of programmed cell death-ligand 1 expression in patients with non-small-cell lung cancer: evidence from an updated meta-analysis. OncoTargets and Therapy. 2015;8:3595-601.

32. Zhou ZJ, Zhan P, Song Y. PD-L1 over-expression and survival in patients with non-small cell lung cancer: a meta-analysis. Transl Lung Cancer Res. 2015;4:203-8

33. Ilie M, Hofman V, Dietel M, Soria JC, Hofman P. Assessment of the PD-L1 status by immunohistochemistry: challenges and perspectives for therapeutic strategies in lung cancer patients. Virchows Arch. 2016.

34. Teixidó C, Karachaliou N, González-Cao M, Morales-Espinosa D, Rosell R. Assays for predicting and monitoring responses to lung cancer immunotherapy. Cancer Biology \& Medicine. 2015;12:87-95.

35. Vansteenkiste J, Fehrenbacher L, Spira A, et al. Atezolizumab monotherapy vs docetaxel in $2 \mathrm{~L} / 3 \mathrm{~L}$ non-small cell lung cancer: Primary analyses for efficacy, safety and predictive biomarkers from a randomized phase II study (POPLAR). Vienna: European Cancer Congress; 2015.

36. Hirsch FR, McElhinny A, Stanforth D, et al. PD-L1 IHC assays for lung cancer: results from phase 1 of the "blueprint PD-L1 assay comparison project". New Orleans: AACR Annual Meeting; 2016.

37. Desai N. Spatiotemporal effects on Programmed Death Ligand 1 (PD-L1) expression and Immunophenotype of Non-small Cell Lung Cancer (NSCLC) (ID1609). Denver: 2015 World Conference on Lung Cancer; 2015.

38. Kowanetz M. Evaluation of PD-L1 expression in metachronous tumor samples and FDG-PET as a predictive biomarker in Ph2 study (FIR) of Atezolizumab (MPDL3280A) (ID2207). Denver: 2015 World Conference on Lung Cancer; 2015.

39. Sheffield BS, Geller G, Pleasance E, et al. Predictive biomarker testing for programmed cell death 1 inhibition in Non-small cell lung cancer (ID 1081). Denver: 2015 World Conference on Lung Cancer; 2015.

40. Mitchell P, Murone C, Asadi K, Harbison CT, Knight S, John T. Programmed Death Ligand-1 (PDL-1) expression in Non-Small Cell Lung Cancer (NSCLC): analysis of a large early stage cohort; and concordance of expression in paired primary-nodal and primary-metastasis tumour samples (ID 3226). Denver: 2015 World Conference on Lung Cancer; 2015.

41. Sheng J, Fang W, Yu J, et al. Expression of programmed death ligand-1 on tumor cells varies pre and post chemotherapy in non-small cell lung cancer. Scientific Reports. 2016;6:20090.

42. Twyman-Saint Victor C, Rech AJ, Maity A, et al. Radiation and dual checkpoint blockade activate non-redundant immune mechanisms in cancer. Nature. 2015;520:373-7.

43. Deng L, Liang $\mathrm{H}$, Burnette $\mathrm{B}$, et al. Irradiation and anti-PD-L1 treatment synergistically promote antitumor immunity in mice. The Journal of Clinical Investigation. 2014;124:687-95.

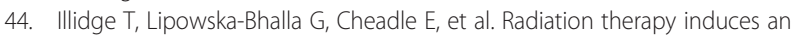
adaptive upregulation of PD-L1 on tumor cells which may limit the efficacy of the anti-tumor immune response but can be circumvented by anti-PDL1. Int J Radiat Oncol Biol Phys. 2014;90:S776.

45. Snyder A, Makarov V, Merghoub T, et al. Genetic basis for clinical response to CTLA-4 blockade in melanoma. N Engl J Med. 2014;371:2189-99.

46. Rizvi NA, Hellmann MD, Snyder A, et al. Cancer immunology. Mutational landscape determines sensitivity to PD-1 blockade in non-small cell lung cancer. Science. 2015;348:124-8.

47. Le DT, Uram JN, Wang H, et al. PD-1 blockade in tumors with mismatchrepair deficiency. New England Journal of Medicine. 2015;372:2509-20.

48. van Gool IC, Eggink FA, Freeman-Mills L, et al. POLE proofreading mutations elicit an antitumor immune response in endometrial cancer. Clin Cancer Res. 2015;21:3347-55.

49. Apetoh L, Ladoire S, Coukos G, Ghiringhelli F. Combining immunotherapy and anticancer agents: the right path to achieve cancer cure? Ann Oncol. 2015;26(9): 1813-823.

50. Bracci L, Schiavoni G, Sistigu A, Belardelli F. Immune-based mechanisms of cytotoxic chemotherapy: implications for the design of novel and rationalebased combined treatments against cancer. Cell Death Differ. 2014;21:15-25.
51. Antonia S, Goldberg SB, Balmanoukian AS, et al. Phase Ib study of MEDI4736, a programmed cell death ligand-1 (PD-L1) antibody, in combination with tremelimumab, a cytotoxic T-lymphocyte-associated protein-4 (CTLA-4) antibody, in patients with advanced NSCLC. J Clin Oncol. 2015;33(suppl):abstr 3014.

52. Antonia S, Gettinger SN, Chow L, et al. Nivolumab (anti-PD-1; BMS-936558, ONO-4538) and ipilimumab in first-line NSCLC: Interim phase I results. J Clin Oncol. 2014;32:suppl; abstr 8023.

53. Antonia S, Goldberg SB, Balmanoukian A, et al. Safety and antitumour activity of durvalumab plus tremelimumab in non-small cell lung cancer: a multicentre, phase 1b study. Lancet Oncol. 2016:17:299-308.

54. Hellmann MD, Gettinger SN, Goldman JW, et al. CheckMate 012: Safety and efficacy of first-line (1 L) nivolumab (nivo; N) and ipilimumab (ipi; I) in advanced (adv) NSCLC (Abstract 3001). J Clin Oncol. 2016;34:suppl; abstr 3001.

55. Bauer TM, McCleod M, Chandler JC, et al. An ongoing phase IIIb/IV safety trial of nivolumab (NIVO) in patients (pts) with advanced or metastatic nonsmall-cell lung cancer (NSCLC) who progressed after receiving 1 or more prior systemic regimens. J Clin Oncol. 2015;33:suppl; abstr 3013.

56. Hodi FS, Sznol M, Kluger HM, et al. Long-term survival of ipilimumab-naive patients (pts) with advanced melanoma (MEL) treated with nivolumab (anti-PD-1, BMS-936558, ONO-4538) in a phase I trial. J Clin Oncol. 2014;32:suppl; abstr 9002.

57. Robert C, Long GV, Brady B, et al. Nivolumab in previously untreated melanoma without BRAF mutation. N Engl J Med. 2015;372:320-30.

58. Weber JS, D'Angelo SP, Minor D, et al. Nivolumab versus chemotherapy in patients with advanced melanoma who progressed after anti-CTLA-4 treatment (CheckMate 037): a randomised, controlled, open-label, phase 3 trial. Lancet Oncol. 2015;16:375-84.

59. Larkin J, Chiarion-Sileni V, Gonzalez R, et al. Combined nivolumab and ipilimumab or Monotherapy in untreated melanoma. New England Journal of Medicine. 2015:373:23-34.

60. Kefford R, Ribas A, Hamid O, et al. Clinical efficacy and correlation with tumor PD-L1 expression in patients (pts) with melanoma (MEL) treated with the anti-PD-1 monoclonal antibody MK-3475. J Clin Oncol 2014;32:suppl; abstr 3005 .

61. Puzanov I, Dummer R, Schachter J, et al. Efficacy based on tumor PD-L1 expression in KEYNOTE-002, a randomized comparison of pembrolizumab (pembro; MK-3475) versus chemotherapy in patients (pts) with ipilimumab-refractory (IPI-R) advanced melanoma (MEL). J Clin Oncol. 2015;33:suppl; abstr 3012.

62. Robert C, Schachter J, Long GV, et al. Pembrolizumab versus ipilimumab in advanced melanoma. New England Journal of Medicine. 2015;372:2521-32.

63. Spigel DR, Chaft JE, Gettinger SN, et al. Clinical activity and safety from a phase II study (FIR) of MPDL3280A (anti-PDL1) in PD-L1-selected patients with nonsmall cell lung cancer (NSCLC). J Clin Oncol. 2015;33:suppl; abstr 8028.

64. Spira A, Park K, Mazieres J, et al. Efficacy, safety and predictive biomarker results from a randomized phase II study comparing MPDL3280A vs docetaxel in 2 L/3L NSCLC (POPLAR). J Clin Oncol. 2015;33:suppl; abstr 8010.

65. Besse $B$, Johnson $M$, Janne $M$, et al. Phase II, single-arm trial (BIRCH) of atezolizumab as first-line or subsequent therapy for locally advanced or metastatic PD-L1-selected non-small cell lung cancer (NSCLC). Vienna: European Cancer Congress; 2015.

66. Powles T, Eder JP, Fine GD, et al. MPDL3280A (anti-PD-L1) treatment leads to clinical activity in metastatic bladder cancer. Nature. 2014;515:558-62.

67. Rosenberg JE, Hoffman-Censits J, Powles T, et al. Atezolizumab in patients with locally advanced and metastatic urothelial carcinoma who have progressed following treatment with platinum-based chemotherapy: a single-arm, multicentre, phase 2 trial. Lancet. 2016;387(10031):1909-920.

68. Rizvi N, Brahmer J, Ou SI, et al. Safety and clinical activity of MEDI4736, an anti-programmed cell death-ligand 1 (PD-L1) antibody, in patients with non-small cell lung cancer (NSCLC). J Clin Oncol. 2015;33:suppl;abstr 8032.

69. Segal NH, Ou SI, Balmanoukian AS, et al. Safety and efficacy of MEDI4736, an anti-PD-L1 antibody, in patients from a squamous cell carcinoma of the head and neck (SCCHN) expansion cohort. J Clin Oncol. 2015;33: suppl; abstr 3011.

70. Gulley JL, Spigel DR, Kelly K, et al. Avelumab (MSB0010718C), an anti-PD-L1 antibody, in advanced NSCLC patients: A phase $1 \mathrm{~b}$, open-label expansion trial in patients progressing after platinum-based chemotherapy. J Clin Oncol. 2015;33:suppl; abstr 8034.

71. Apolo AB, Infante JR, Hamid O, et al. Safety, clinical activity, and PD-L1 expression of avelumab (MSB0010718C), an anti-PD-L1 antibody, in patients with metastatic urothelial carcinoma from the JAVELIN Solid Tumor phase Ib trial. J Clin Oncol. 2015;33:suppl 2S; abstr 367. 